\title{
Management of severe malnutrition
}

\section{Abstract}

In summary, if the Millenium Development Goals (MDGs) are to be addressed effectively, it is imperative that severe malnutrition be addressed at the international and national level, using strategies that work, and that additional resources are committed to improving hospital treatment and establishing community-based rehabilitation programmes. ${ }^{20}$ Many gaps still exist in our knowledge regarding the management of severe malnutrition at all levels. Until such time when research can fill these gaps, we will have to continue to use what guidelines have shown to produce positive results.

S Afr J Clin Nutr 2010;23(1) Supplement:S22-S24

Severe malnutrition is defined as a severe wasting $(<70 \%$ weight for length or $<-3$ Z-score) and/or oedema. ${ }^{1}$ The global estimate of wasting is approximately 10\% (approx 55 million children), with severe wasting at 3,5\% (approximately 19 million children). ${ }^{2}$ Stunting, however, is regarded as a problem of greater magnitude than underweight or wasting, as it more accurately reflects nutritional deficiencies and illness that occur during the most critical period of development and growth. ${ }^{3}$ Twenty four countries account for more than $80 \%$ of the worldwide burden of chronic undernutrition (i.e. stunting). South Africa is counted amongst one of these countries. ${ }^{3}$ It is not surprising that stunting is highest for the poorest segments of the population within countries. ${ }^{2}$ Stunting affects approximately 195 million children under the age of five years. ${ }^{3}$ Malnutrition is an underlying factor in approximately $50 \%$ of the nearly 10 million deaths in children under the age of five years from preventable causes. ${ }^{4}$

Serious attention needs to be paid to the long-term effects and burden of undernutrition. The child is at its most vulnerable during the period of most rapid growth and development (this includes the period of significant brain formation). ${ }^{2,3}$ Chronic undernutrition results in diminished cognitive and physical development. ${ }^{3}$ Early growth failure (within the first two years of life) is associated with reduced adult stature, which in turn is associated with lower educational achievement as well as lower economic status in adulthood through reduced productivity and income earning capacity. ${ }^{3,5}$ The situation is further exacerbated by the increased risk of chronic disease in adulthood in children who had deficient growth before the age of two years, but experienced rapid weight gain in later stages of childhood. $^{3}$

Globally, progress toward achieving the Millenium Development Goals (MDG) has been varied, with some countries, most of them in sub-Saharan Africa, actually worsening in terms of the numbers of children who are underweight. The stunting prevalence has not decreased in the sub-Saharan Africa since the mid-1990s, with
South Africa being one of these countries according to UNICEF., Furthermore, South Africa has shown an increased trend since 1990 in the mortality of children under the age of 5 years. ${ }^{8}$

There is, however, difference in the statistics given by UNICEF and the National Food Consumption Survey. From the NFCS there appears to be a slight decline in stunting from 1994 to 2005 at $23 \%$ and $18 \%$ respectively. In the Western Cape stunting has remained static at around $12 \%$. However, it would appear that the prevalence of wasting over the same period has increased slightly from 3 to 4,5 nationally and alarmingly from 1 to $11,5 \%$ in the Western Cape in the period from 1994 to $2005 .{ }^{9}$

Annually large amounts of money are invested to improve nutritional outcomes in poor countries by the international community. Approximately \$250-300 million a year for the first half of this decade was invested in basic nutrition. This, however, translates to just over \$2 a year per infant for the 130 million infants under two years who could benefit from the investment. This is less than the estimated \$5-10 per child which effective large scale community nutrition programmes should be spending. Interestingly HIV/AIDS receives $\$ 2.2$ billion per year in foreign aid, although it contributes less to "disability adjusted life years" than does child undernutrition. The serious consequences of undernutrition are not well understood at "high level".

\section{National policies}

To achieve the first MDG target depends to a great extent on the effective implementation of large scale nutrition and health programmes that will provide appropriate food, health and care for all children within a country. ${ }^{3}$

The Lancet series ${ }^{10}$ reviewed the national strategies that have been proven to work. The interventions that had the greatest impact on reducing malnutrition and mortality were: iron supplementation, salt iodisation, vitamin A supplementation for children aged 6-59 
months, breastfeeding strategies based on individual and group counselling, zinc supplementation in the management of diarrhoea, fortification of staple foods, behaviour change, communication to improve complementary feeding and interventions to improve hygiene practices. ${ }^{3,10}$

Certain actions have been found to be ineffective in reducing undernutriton such as growth monitoring (unless it is linked to adequate nutrition counselling and referral), preschool feeding programmes targeting children older than 24 months of age and school feeding programmes targeting children older than five years of age. This last strategy may, however, have non-nutritional benefits for education. ${ }^{10}$

Though not all effective strategies can be generalised for all countries, a sizeable effect can be achieved with high coverage of four or five proven interventions. ${ }^{11}$ However, for national strategies to be effective, commitment is required from all the country's roleplayers. In spite of the dire statistics, nutrition remains a low priority on the national development agendas of many countries and often has no clear institutional home..$^{3,11}$

Undernutrition often remains unnoticed until it has reached a severe level. Children may appear healthy even whilst facing the grave risks associated with undernutrition. Policy makers often do not understand the urgency of the situation and may not understand how improved nutrition contributes to long-term national, social and economic goals. $^{3}$

In many cases, national programmes do not fully succeed due to the lack of political commitment (defined as the allocation of human, financial and organisational resources). ${ }^{11}$ National leaders and those who control national financial resources are more likely to be more successful in promoting nutrition strategies than public health advocates or technical staff from donor or UN agencies. Nutrition goals therefore should be included in all appropriate government sectors and their policies and operations. National sectors, other than Health, may also have an impact on nutritional status e.g. economic policies to address poverty, trade and agriculture, opportunities that are often under-exploited. ${ }^{11}$

However, political commitment to nutrition will also only result in improvements in nutritional status if supported by interventions that are effective and that are able to be implemented at high and sustainable levels of coverage. Historical evidence has shown that the nutrition of mothers and children can be improved relatively quickly, given the combined effort politically, strategic planning and adequate resources. ${ }^{11}$

Paradoxically, South Africa has poor health outputs and outcomes despite relatively high health expenditure and many supportive policies. The poor successes of policies are often due to the failure to build capacity to implement the policies and programmes and to monitor implementation. A great deal of attention and resources (including medical personnel) have been absorbed by a few health programmes, e.g. HIV programmes. South Africa's commitment to the MDGs is being crippled by the relentless rise in the burden of diseases which is leading to a vicious cycle of increased expenditure, distorted responses by the health system, out-migration of human resources and increased social issues. ${ }^{12}$

The difficulties in implementing effective national strategies successfully is further exacerbated by an international nutrition system that has little respect for country generated plans, local timetables or political processes of a country. ${ }^{11}$

\section{International actions}

International initiatives often do not have the desired effects as they often behave adversarially and compete for attention. They also often siphon off scarce human resources and promote poorly designed solutions to problems that they cannot solve on their own. The function of international initiatives should be to directly support national structures in high burden countries and to produce "global public goods" e.g. setting standards and identifying priorities; mobilising, pooling and distributing financial resources; providing nutrition services where national sectors are unable or unwilling to do so themselves; strengthening human and institutional resources through training and capacity building and finally research and development. $^{7}$

The international community devotes few resources to nutritionrelated organisational development in low and middle income countries. Many of the major international organisations seem to lack appropriately skilled staff at central level.

Efforts to manage undernutrition internationally have up to date mostly been fragmented. The Lancet series recommended that all interested parties working to eliminate undernutrition should come together to review the structures that are currently in place; at how to simplify the current system; how to strengthen regional and subregional networks; and to develop a strategy to prioritise topics for research. ${ }^{7}$ The path forward, from projects and evidence from randomised controlled trials to developing large scale programmes with sound design to achieve results, needs to be identified. ${ }^{6}$

\section{Facility-based management of severe malnutrition}

Probably the best known international initiative for the management of severe acute malnutrition is the WHO guideline, Ten Steps for the management of severe malnutrition; promoted as the standard by which severely malnourished children should be treated..$^{13,14}$

The literature indicates that if implemented correctly, the protocol can improve case fatality rates from around $40 \%$ to the order of $1-5 \%{ }^{4}$

When reviewing the evidence on which the Ten Steps are based, it will be found that many studies were conducted in the 1960s and 1970s where it became clear that high protein and fluid intakes $>150 \mathrm{ml} / \mathrm{kg}$ during the initial phase increased mortality, and that careful feeding regimens initially increasing in volume and density toward a high energy formula to aid rapid catch up growth, reduced mortality. ${ }^{15}$ These studies eventually led to the development of the WHO protocol.

In 2006 Brewster published a critical review of the evidence on which the WHO guideline is based. The review highlights a number of aspects where the WHO guidelines may be lacking in evidence and which may be important to consider. He pointed out that there is a lack of evidence that the high mortality rates are directly proportional to the quality of care and that the severity of illness is also largely a contributing factor. He also suggests that a protocol alone is not sufficient in adequately managing severe malnutrition, but that an experienced health worker made better clinical judgments than one who follows a protocol. ${ }^{16}$ There appears to be support for recommendations made by the WHO on the necessity of routine 
empirical antibiotic treatment, the prevention of hypoglycaemia through small frequent feeds, zinc supplementation, delayed iron supplementation, but that it may be preferable to provide smaller daily doses of vitamin $A$ than a single large dose and that children with kwashiorkor may require more potassium than recommended. ${ }^{16,15}$

The F75 and F100 formulas as recommended by the WHO must also be evaluated critically. These formulae have a number of apparent shortcomings that need to be addressed. Both formulae have high osmolalities, namely 333 and $419 \mathrm{~m} 0 \mathrm{sml} / \mathrm{l}$ and lactose contents of 13 and $42 \mathrm{~g} / \mathrm{l}$ respectively. ${ }^{15}$ The importance of lactose intolerance in severely malnourished children tends to be downplayed. Enteropathy is often a feature of severe malnutrition and is usually accompanied with clinically significant carbohydrate intolerance with osmotic diarrhoea. ${ }^{17}$

It is uncertain what the rationale is for the very low protein content of the F 75 formula at only $4,8 \%$ of energy as protein. An implication of the very low protein content is that the phosphorous requirements recommended by the WHO are not met. ${ }^{15,17}$

Another matter for concern in many countries with a high burden of severely malnourished children is the poor accessibility to the commercially F75 and F100 diets recommended by the WHO. So health facilities need to mix their own formulae and have to add their own mineral and vitamin mixes. ${ }^{15} \mathrm{~A}$ matter that is not being considered, is the risk of nosocomial and food-related infections when milk formulae are prepared. In a study conducted in 2008 in 18 public health facilities in South Africa by the DOH, it was found that a vast majority of the samples of powdered infant formulae tested positive for pathogens after preparation, indicating inadequate hygiene during feed preparation..$^{18}$ It only stands to reason that a feed that requires a greater variety of constituents will run an even greater risk of being contaminated during preparation. Another shortcoming of mixing the formula from the recipe is that not all facilities have access to the vitamin and mineral mixes required to make up the micronutrient content. There is therefore a need for an affordable, hygienic and nutritional adequate alternative to the F75 and $\mathrm{F} 100$ recipes.

Brewster ${ }^{17}$ pointed out that there are shortcomings in the guidelines in that the management of "marasmus" vs "kwashiorkor"; HIV negative and positive children; and teaching hospitals and rural hospitals are all "lumped" together. The WHO has confirmed that gaps in knowledge remain, particularly in the feeding of very young infants and also infants living with HIV/AIDS. ${ }^{19}$

\section{Community-based therapeutic care}

The area in the management of severe malnutrition that is enjoying considerable amount of attention is community-based therapeutic care.

Community-based care refers to treatment that is implemented at home with some external input such as a health worker. ${ }^{20}$ Motivations for implementation of community-based therapeutic care include factors such as hospital budgetary constraints, bed shortages, the reduction of hospital-acquired infections and requests by families for early discharge due to concern for the care of other family members or loss of earnings..$^{20}$ One disadvantage to community-based care, is that the weight gain is often less than what is achieved during hospitalisation.
Community-based treatment occurs once the initial stabilisation phase during hospitalisation is completed. The initial stabilisation usually lasts two to seven days. Thereafter there are three main options for community-based care, namely: short-stay day care, rehabilitation at home with clinic visits, or rehabilitation at home with Ready to Use Therapeutic Foods (RUTF). There are advantages and disadvantages to all three option, and the option best suited to the situation should be considered. ${ }^{20}$ The international trend seems to be toward the third option of rehabilitation at home with RUTF. The RUTF is a paste where part of the skim milk is replaced with a groundnut paste. It is more energy dense than the F100 formula, but has a similar nutrient to energy density ratio. ${ }^{15,20}$ The advantages of the RUTF is that it does not support bacterial growth, can be consumed without the addition of water or processing, it is energy dense without the high osmolarity problem..$^{15}$ There is also some evidence that the RUTF supports better weight gain than the F100. ${ }^{15,20}$ Limitations however to the RUTF are the high cost of the product, sharing of the product with other family members, compensatory reductions of home food and poor compliance. ${ }^{15}$

Community-based care will however only be effective if the infrastructure and adequate resources (including nutrition educators) are in place to provide the support to the mothers and caregivers after discharge. The mother or caregiver requires training on the importance of adequate feeding and rehabilitation at home and the child's monitoring either through home visits or at the clinic. ${ }^{20}$ Therefore, optimal community-based rehabilitation requires careful planning and cannot be implemented without considerable initial inputs and resources being in place..$^{20}$

\section{References:}

. Briend A, Prudhon C, Prinzo ZW, Daelmans B, Mason JB. Putting the management of severe malnutrition back on the international health agenda. Food and Nutrition Bulletin 2006;27(3)supplement:S3-S6.

. Black RE,Allen LH, Bhutta ZA, et al. Maternal and Child Undernutrition 1. Maternal and child undernutrition: global and regional exposures and health consequences. Lancet 2008:371:243-260.

3. UNICEF. Tracking Progress on Child and Maternal Nutrition [Online] 2009 [Access 2009, December 22]; Available: $h$ http://www.unicef.org/publications/index.html.

4. Collins S, Sadler K, Dent N, et al. Key issues in the success of community-based management of severe malnutrition. Food and Nutrition Bulletin 2006;27(3)supplement:S49-70.

5. Victora CG, Adair L, Fall C, et al. Maternal and Child Undernutrition 2: Maternal and Child Undernutrition: consequences for adult health and human capital. Lancet 2008;371:340-357.

6. Schultink W, Arabi M Effective Nutrition programming for children and the role of UNICEF: Conensus point from an expert Consultation. Food Nutr Bull 2009;30(2):189-196.

7. Morris SS; Cogill B; Uauy R. Maternal and Child Undernutrition 5: Effective international action against undernutrition: why has it proven so difficult and what can be done to accelerate progress? Lancet 2008;371:608-621.

8. Kleinert S \& Horton R. South Africa's Health: departing for a better future? Lancet 25 August 2009 (published online)

9. Draft Report: The nutritional status of children in the Western Cape (October 2009). University of Western Cape \& Western Cape Department of Health (Unpublished data).

10. Bhutta Z, Ahmed T, Black RE, et al. Maternal and Child Undernutrition 3: What works? Interventions for maternal and child undernutrition and survival. Lancet 2009;371:417-440.

11. Bryce J, Coitinho D, Darnton-Hill I, Pelletier D, Pinstrup-Anderson P. Maternal and Child Undernutrition 4 : Maternal and child undernutrition: effective action at national level. Lancet 2008; 371:510-526.

12. Chopra M, Lawn JE, Sanders D, et al. Achieving the Health Millennium Development Goals for South Africa: challenges and priorities. Lancet 25 August 2009 (published online).

13. WHO. Management of severe malnutrition: a manual for physicians and other senior health worker. [Online] 1999 [Access 2009, December 15]; Available: http://www.who.int/nutrition/publications/ malnutrition/en/

14. WH0. Guidelines for the inpatienttreatment of severely malnourished children. [Online] 2003 [Access 2009, December 15]; Available: http://www.who.int/nutrition/publications/severemalnutrition/9241546093/ en/index.html.

15. Brewster D. Critical appraisal of the management of severe malnutrition:2. Dietary management. Jn Paed Child Health 2006;42:575-582.

16. Brewster D. Critical appraisal of the management of severe malnutrition: 1. Epidemiology and treatment guidelines. Jnl Paed Child Health 2006;42:568-574.

17. Brewster D. Critical appraisal of the management of severe malnutrition:3 Complications. JnI Paed Child Health 2006;42:583-593.

18. Guidelines On Safe Preparation, Storage And Handling Of Powdered Infant Formula in Health Facilities And Home Environment. Draft 4 Department of Health 2009.

19. WHO Severe malnutrition: Report of a consultation to review current literature. [Online] 2004 [Access 2009, December 15]; Available: http://www.who.int/nutrition/publications/severemalnutrition/9241593318/ en/index.html.

20. Ashworth A. Efficacy and effectiveness of community-based treatment of severe malnutrition. Food and Nutrition Bulletin 2006;27(3)supplement:S24-48. 